\title{
Peginterferon alpha-based therapy for chronic hepatitis B focusing on HBsAg clearance or seroconversion: a meta-analysis of controlled clinical trials
}

Wen-cong $\mathrm{Li}^{1+}$, Mao-rong Wang ${ }^{2 \dagger}$, Ling-bo Kong ${ }^{1+}$, Wei-guang Ren ${ }^{1+}$, Yu-guo Zhang ${ }^{1+}$ and Yue-min Nan ${ }^{1 *}$

\begin{abstract}
Background: Interferon alpha (IFNa) therapy has been widely used in the treatment of chronic hepatitis B (CHB) for decades. Nucleos(t)ide analogues are also increasingly used to treat CHB recently. More and more studies are being carried out concerning the clearance or seroconversion of HBsAg, which is recognized as an ideal goal of CHB therapy. This study conducted a meta-analysis to estimate the effect of pegylated interferon alpha (peginterferon $\alpha$, PEG-IFN $\alpha$ )-based therapy on HBsAg clearance or seroconversion in CHB.
\end{abstract}

Methods: All available controlled clinical trials, published from 2004 to 2010, with the following antiviral therapies for CHB patients: PEG-IFN $\alpha$ combined with lamivudine (LAM), PEG-IFN $\alpha$ only, conventional IFN $\alpha$ and LAM, with a course $\geq 24$ weeks, were meta-analysed for HBsAg clearance and seroconversion.

Results: Fourteen trials (involving a total of 2,682 patients) were identified, including seven high-quality and seven low-quality studies. The analysis results of the different antiviral therapies on HBsAg clearance or seroconversion were as follows: 1. No significant difference in HBsAg clearance or seroconversion was observed between the combination therapy group and PEG-IFN $\alpha$ monotherapy group [odds ratio (OR) $=1.16,95 \%$ confidence intervals (Cl) (0.73-1.85), $P=0.54$ and $\mathrm{OR}=1.07,95 \% \mathrm{Cl}(0.58-1.97), P=0.82$, respectively]; 2 . HBsAg clearance and seroconversion rates in patients with combination therapy were markedly higher than in those with LAM monotherapy $[\mathrm{OR}=9.41,95 \% \mathrm{Cl}(1.18-74.94), P=0.03$, and $\mathrm{OR}=12.37,95 \% \mathrm{Cl}(1.60-95.44), P=0.02$, respectively]; 3. There was significant difference in HBsAg clearance between the PEG-IFNa group and IFNa monotherapy group $[O R=4.95,95 \% \mathrm{Cl}(1.23-20.00), P=0.02]$, but not in seroconversion $[\mathrm{OR}=2.44,95 \% \mathrm{Cl}(0.35-17.08), P=0.37] ; 4$. PEG-IFNa was superior to LAM in HBsAg seroconversion [OR $=14.59,95 \% \mathrm{Cl}(1.91-111.49), P=0.01]$.

Conclusions: PEG-IFNa facilitated HBsAg clearance or seroconversion in CHB patients. PEG-IFNa-based therapy was more effective than LAM monotherapy in achieving HBsAg clearance or seroconversion for both HBeAg-positive and HBeAg-negative CHB patients. There was no significant difference in HBsAg clearance or seroconversion between PEG-IFNa/LAM combination therapy and PEG-IFNa monotherapy. PEG-IFNa was obviously superior to conventional IFNa in HBsAg clearance, but not in HBsAg seroconversion. Although PEG-IFNa produced significantly higher rates of $\mathrm{HBsAg}$ clearance and seroconversion, the absolute change in the proportion of HBsAg clearance and seroconversion was low (about 3-6\%). Therefore, additional interventions are needed to improve the rate of positive outcomes.

Keywords: hepatitis B, HBsAg, peginterferon, interferon, lamivudine

\footnotetext{
* Correspondence: nanyuemin@163.com

+ Contributed equally

'Department of Traditional and Western Medical Hepatology, Third Hospital

of Hebei Medical University, Shijiazhuang, China

Full list of author information is available at the end of the article
} 


\section{Background}

Antiviral therapy has been recognized as the first choice for chronic hepatitis $\mathrm{B}(\mathrm{CHB})$ treatment and approved as an efficient approach to ameliorating hepatic inflammation and fibrosis, further preventing liver cirrhosis and hepatocellular carcinoma [1-4]. The state of hepatitis $B$ virus (HBV) DNA $<2,000 \mathrm{IU} / \mathrm{mL}$ and alanine aminotransferase (ALT) normalization after treatment is a good prediction in both $\mathrm{HBeAg}$-positive and $\mathrm{HBeAg}$-negative $\mathrm{CHB}$ [5-9]. Nevertheless, HBsAg clearance and seroconversion, characterized by the loss of serum HBsAg with or without anti-HBs antibody development, are the main markers of a successful immunological response to HBV infection and the closest outcome to clinical cure [10-14]. Some researches indicated that interferon-based therapy, especially pegylated interferon alpha (peginterferon $\alpha$, PEGIFN $\alpha$ ), obviously outstripped nucleos(t)ide analogues in achieving HBsAg clearance and seroconversion [15-23]. The weekly administration of PEG-IFN $\alpha$ is likely to improve patients' compliance rate, while obtains much better pharmacokinetics [24]. Therefore, several practice guidelines for the management of $\mathrm{CHB}$ have considered PEG-IFN $\alpha$ as a first-line therapy for $\mathrm{CHB}$ patients [25,26]. However, it remains unclear whether the rate of $\mathrm{HBsAg}$ clearance and seroconversion is higher in $\mathrm{CHB}$ patients receiving PEG-IFNa-based therapy than in those receiving conventional IFN $\alpha$ or nucleos(t)ide analogues.

In this study, we aimed to elucidate the efficacy of PEG-IFN $\alpha$-based therapy in the treatment of CHB. Furthermore, our analysis also focused on the rates of HBsAg clearance and seroconversion, which might benefit to optimize the antiviral treatments for $\mathrm{CHB}$. We restricted the comparison groups to PEG-IFN $\alpha$-based therapy and conventional IFN $\alpha$ or lamivudine (LAM) treatments due to no available controlled clinical trials of PEG-IFN $\alpha$-based therapy compared with nucleos $(t)$ ide analogues except LAM.

\section{Methods}

\section{Literature retrieval and study design}

Two researchers independently operated the literature retrieval, trial selection and data extraction, reaching to consensus by conferring with each other when discrepancies appeared. The researchers performed a systemic literature retrieval using electronic databases including PubMed (1966-2010), English medical Current Contents (EMCC 1995-2010), China National Knowledge Infrastructure (CNKI 1979-2010), China Hospital Knowledge Database (CHKD 1994-2010) and the Cochrane library clinical trials registry (Issue 3 of 4, Jul 2010). The retrieval was finished in November 2010. The following keywords were used: 'hepatitis B', 'peginterferon', 'pegylated interferon', and 'HBsAg'. In addition, a manual search was conducted using citations in previous publications.
The included studies were divided into different groups according to intervention treatments. The prognosis of the patients were recorded and analysed. Data were extracted by study methodology and defined efficacy measures. Only data regarding the regimens in question were extracted, while data concerning other regimens were also reviewed, and if they were found to be of significance to our study, were noted and discussed. Separate meta-analyses were performed for each group.

\section{Criteria for inclusion and exclusion}

The inclusion criteria were as follows: (i) study design: controlled clinical trials; (ii) study population: $\mathrm{CHB}$ patients; (iii) intervention: PEG-IFN $\alpha$ combined with LAM therapy versus PEG-IFN $\alpha$ or LAM monotherapy, PEG-IFN $\alpha$ versus IFN $\alpha$ or LAM monotherapy; (iv) outcomes: HBsAg clearance or seroconversion.

The exclusion criteria were as follows: (i) study design: non-clinical studies; (ii) study population: non-adult population, women with pregnancy or lactation, patients received liver transplantation, patients co-infected with hepatitis $C$ virus, hepatitis D virus or human immunodeficiency virus, patients with a history of alcohol or drug abuse, hepatocellular carcinoma, decompensated liver disease, serious medical or psychiatric illness; (iii) intervention: concurrently using corticosteroid, immunosuppressive agents or Chinese herbal medicine; (iv) outcomes: not reporting any of the efficacy measures of HBsAg clearance or seroconversion as defined by the authors; (v) republished studies, or the full text were not available. Our search was restricted by language; citations in languages other than English or Chinese were not included.

\section{Efficacy measures and definitions}

HBsAg clearance was defined as the disappearance of HBsAg from the serum. HBsAg seroconversion was defined as HBsAg disappearance and anti-HBs antibody appearance.

\section{Study quality and homogeneity}

Methodological quality of the included studies was assessed according to the Jadad quality scale [27], an established composite score evaluating randomization, concealment and reporting of patient withdrawal and dropout rates, with more than or equal to 3 scores defined as high-quality. We performed a sensitivity analysis of quality by considering all studies to be of highquality. Heterogeneity was assessed for each analysis.

\section{Statistical analyses}

Quantitative meta-analyses were performed to assess differences between PEG-IFN $\alpha$-based therapy and IFN $\alpha$ or LAM monotherapy groups. Statistical analysis was 
performed and the Forest plots were generated using the software of Review Manager (RevMan 5.0.24.0, the Nordic Cochrane Center, Rigshospitalet). The odds ratios (OR) were calculated along with their respective 95\% confidence intervals (CI) and presented for each individual study. If a clinical trial has no subject (0\%) developing the outcome of concern in either of the two comparison groups, we just input the "zero" to the computer, then it will add 0.5 to each of the four cells in the $2 \times 2$ table by RevMan 5.0.24.0 automatically. Statistical heterogeneity between trials was evaluated by the chisquare $\left(\chi^{2}\right)$ and I square $\left(\mathrm{I}^{2}\right)$ tests, with significance being set at $P<0.10$. In the absence of statistically significant heterogeneity, the fixed-effect method was used to combine the results. When heterogeneity was confirmed ( $P=0.10$ or lower), the random-effect method was used. Publication bias was assessed by funnel plots.

\section{Results}

\section{Study selection and characteristics}

Our electronic and manual searches identified 518 articles. Thirty-three potentially eligible controlled clinical trials using PEG-IFN $\alpha$-based therapy for chronic HBV infection were selected, of which nineteen were excluded. Seventeen were duplicate publications, the other two trials were excluded because the interventions contained different adefovir dipivoxil-based therapy and could not be analyzed using meta-analysis for the marked heterogeneity with the included trials. The flow diagram was shown in Figure 1. Therefore, fourteen trials involving a total of 2,682 patients were analyzed in this study. Among these trials, five presented both HBsAg clearance and seroconversion [16,21,22,28,29], eight displayed HBsAg clearance [17-20,23,30-32] and one showed HBsAg seroconversion [15]. Three trials

$$
\begin{aligned}
& \text { Studies retrieved by literature search }(\mathrm{n}=518) \\
& \text { Studies excluded: did not meet inclusion criteria }(n=386) \\
& \checkmark \\
& \text { Included studies by reading titles and abstract }(\mathrm{n}=132) \\
& \text { 1. Republished studies }(\mathrm{n}=25) \\
& \text { 2. Did not report HBsAg clearance or seroconversion }(n=67) \\
& \text { 3. Without full text }(n=7) \\
& \text { Included studies by reading full text }(\mathrm{n}=33) \\
& \begin{array}{|l|l|}
\text { 1. Excluded in meta-analysis }(\mathrm{n}=2) \\
\text { 2. Same studies }(\mathrm{n}=17)
\end{array}
\end{aligned}
$$

Figure 1 Literature search and data extraction 
were conducted for long-term follow-up (average 3 years) $[17,19,30]$, the others for $0-72$ weeks $[15,16,18,20-23,28,29,31,32]$. Patients from eleven trials were treated for 48 to 60 weeks [15-20,22,28,30-32], and the rest were treated for 24 weeks $[21,23,29]$. Of these studies, seven were high-quality and seven were lowquality (Jadad scores of 3-5 and Jadad scores $<3$, respectively). All studies were published as full publications, with eight in English [15-19,28,30,31] and six in Chinese [20-23,29,32]. Four studies used sequential therapy $[18,19,29,32]$, and six studies used concurrent therapy $[15-17,28,30,31]$ [Tables 1 and 2].

HBsAg clearance and seroconversion in patients receiving combination therapy or monotherapies PEG-IFN $\alpha+L A M$ combination therapy vs. PEG-IFN $\alpha$ monotherapy

The rates of HBsAg clearance and seroconversion were $6.8 \%$ and $3.7 \%$ in the combination therapy group, and
$6.0 \%$ and $3.5 \%$ in the PEG-IFN $\alpha$ monotherapy group, respectively. The fixed effect model for meta-analysis was used according to the heterogeneity test $\left(\chi^{2}=4.05, \mathrm{df}=\right.$ 6, $P=0.67, \mathrm{I}^{2}=0 \%$ and $\chi^{2}=1.29, \mathrm{df}=3, P=0.73, \mathrm{I}^{2}=$ $0 \%$, respectively). The difference between the two groups did not show statistical significance neither in HBsAg clearance rate $[\mathrm{OR}=1.16,95 \% \mathrm{CI}(0.73-1.85), P=0.54$ ] nor in seroconversion rate $[\mathrm{OR}=1.07,95 \% \mathrm{CI}(0.58$ $1.97), P=0.82$ ] [Figure 2]. We also conducted a sensitivity analysis including high-quality studies only and obtained the same result as the overall trials [Figure 3].

PEG-IFN $\alpha+L A M$ combination therapy vs. LAM monotherapy In comparison with the LAM monotherapy, the combination therapy led to higher HBsAg clearance rates during follow-up $(2.6 \%$ vs. $0 \%$ at 24 weeks, and $6.2 \%$ vs. $0 \%$ at 3 years, respectively). Thus, PEG-IFN $\alpha$ combined with LAM therapy was significantly more effective than LAM monotherapy in HBsAg eradication, especially in long-term follow-ups [OR = 7.24, 95\% CI (0.88-59.28),

Table 1 Characteristics of studies included in the meta-analysis

\begin{tabular}{|c|c|c|c|c|c|c|}
\hline Study & $\mathrm{n}$ & $\begin{array}{l}\text { Study } \\
\text { design }\end{array}$ & $\begin{array}{l}\text { Jadad } \\
\text { score }\end{array}$ & $\begin{array}{l}\text { Therapy } \\
\text { period }\end{array}$ & $\begin{array}{l}\text { Follow-up } \\
\text { period }\end{array}$ & Therapy regimen \\
\hline $\begin{array}{l}\text { Lau G.K.K. } \\
\qquad 2005\end{array}$ & 814 & RCT & 4 & $48 w$ & $24 w$ & $\begin{array}{l}\text { 1. PEG-IFNa-2a }(180 \mathrm{ug} / \mathrm{w})+\text { placebo } \\
\text { 2. PEG-IFNo-2a }(180 \mathrm{ug} / \mathrm{w})+\text { LAM }(100 \mathrm{mg} / \mathrm{d}) \\
\text { 3. LAM }(100 \mathrm{mg} / \mathrm{d})\end{array}$ \\
\hline $\begin{array}{l}\text { Marcellin P. } \\
\quad 2004\end{array}$ & 537 & RCT & 4 & $48 w$ & $24 w$ & $\begin{array}{l}\text { 1. PEG-IFNa-2a }(180 \mathrm{ug} / \mathrm{w})+\text { placebo } \\
\text { 2. PEG-IFNo-2a }(180 \mathrm{ug} / \mathrm{w})+\text { LAM }(100 \mathrm{mg} / \mathrm{d}) \\
\text { 3. LAM }(100 \mathrm{mg} / \mathrm{d})\end{array}$ \\
\hline $\begin{array}{l}\text { Marcellin P. } \\
\quad 2009\end{array}$ & 315 & RCT & 4 & $48 w$ & $3 y$ & $\begin{array}{l}\text { 1. PEG-IFNo-2a }(180 \mathrm{ug} / \mathrm{w})+\text { placebo } \\
\text { 2. PEG-IFNo-2a }(180 \mathrm{ug} / \mathrm{w})+\text { LAM }(100 \mathrm{mg} / \mathrm{d}) \\
\text { 3. LAM }(100 \mathrm{mg} / \mathrm{d})\end{array}$ \\
\hline $\begin{array}{c}\text { Chan H.L.Y. } \\
2005\end{array}$ & 100 & RCT & 3 & $52-60 w$ & $24 w$ & $\begin{array}{l}\text { 1. PEG-IFNa-2b }(1.5 \mathrm{ug} / \mathrm{kg}, 1 / \mathrm{w}, \mathrm{Wt}<65 \mathrm{~kg} ; 100 \mathrm{ug} / \mathrm{w}, \mathrm{Wt} \geq 65 \mathrm{~kg}) 8 \mathrm{w} \rightarrow \\
\text { PEG-IFNa-2b }+ \text { LAM } 24 \mathrm{w} \rightarrow \text { LAM } 28 \mathrm{w} \\
\text { 2. LAM }(100 \mathrm{mg} / \mathrm{d})\end{array}$ \\
\hline $\begin{array}{l}\text { Chan H.L.Y. } \\
2005\end{array}$ & 95 & RCT & 3 & $52-60 w$ & $3 y$ & $\begin{array}{l}\text { 1. PEG-IFNa-2b }(1.5 \mathrm{ug} / \mathrm{kg}, 1 / \mathrm{w}, \mathrm{Wt}<65 \mathrm{~kg} ; 100 \mathrm{ug} / \mathrm{w}, \mathrm{Wt} \geq 65 \mathrm{~kg}) 8 \mathrm{w} \rightarrow \\
\text { PEG-IFNa-2b }+ \text { LAM } 24 \mathrm{w} \rightarrow \text { LAM } 28 \mathrm{w} \\
\text { 2. LAM }(100 \mathrm{mg} / \mathrm{d})\end{array}$ \\
\hline $\begin{array}{l}\text { Janssen HLA } \\
2005\end{array}$ & 266 & RCT & 5 & $52 w$ & $26 w$ & $\begin{array}{l}\text { 1. PEG-IFN } \alpha-2 b(100 \mathrm{ug} / \mathrm{w})+\text { placebo } \\
\text { 2. PEG-IFNa-2b }(100 \mathrm{ug} / \mathrm{w})+\text { LAM }(100 \mathrm{mg} / \mathrm{d})\end{array}$ \\
\hline $\begin{array}{l}\text { Buster E.H.C.J } \\
\quad 2008\end{array}$ & 172 & RCT & 5 & $52 w$ & $3.0 \pm 0.8 y$ & $\begin{array}{l}\text { 1. PEG-IFNa-2b }(100 \mathrm{ug} / \mathrm{w})+\text { placebo } \\
\text { 2. PEG-IFNo-2b }(100 \mathrm{ug} / \mathrm{w})+\text { LAM }(100 \mathrm{mg} / \mathrm{d})\end{array}$ \\
\hline $\begin{array}{l}\text { Kaymakoglu } \\
\text { S. } 2007\end{array}$ & 48 & RCT & 2 & $48 w$ & $24 w$ & $\begin{array}{l}\text { 1. PEG-IFNa-2b }(1.5 \mathrm{ug} / \mathrm{kg}, 1 / \mathrm{w}) \\
\text { 2. PEG-IFNa-2b }(1.5 \mathrm{ug} / \mathrm{kg}, 1 / \mathrm{w})+\text { LAM }(100 \mathrm{mg} / \mathrm{d})\end{array}$ \\
\hline Tian YL 2007 & 72 & RCT & 2 & $48 w$ & $72 w$ & $\begin{array}{l}\text { 1. PEG-IFNa-2a }(180 \mathrm{ug} / \mathrm{w}) \\
\text { 2. Conventional IFNa-2a }(5 \mathrm{MU}, 1 / 2 \mathrm{~d})\end{array}$ \\
\hline $\begin{array}{l}\text { Huang ZL } \\
2010\end{array}$ & 50 & $\mathrm{NRCT}$ & 1 & $52 w$ & 0 & 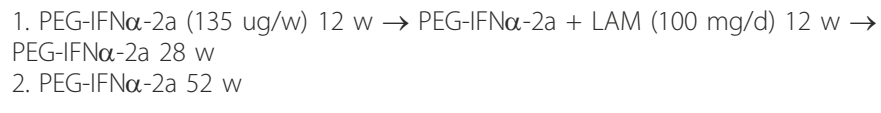 \\
\hline Cui JJ 2006 & 80 & RCT & 2 & $6 \mathrm{~m}$ & $6 \mathrm{~m}$ & $\begin{array}{l}\text { 1. PEG-IFNa-2a (180 ug/w) } \\
\text { 2. Conventional IFN }-2 a(5 \mathrm{MU}, 3 / \mathrm{w})\end{array}$ \\
\hline Li ZQ 2010 & 74 & $\mathrm{NRCT}$ & 1 & $48 w$ & $24 w$ & $\begin{array}{l}\text { 1. PEG-IFN } \alpha-2 \mathrm{a}(180 \mathrm{ug} / \mathrm{w}) \\
\text { 2. Conventional IFN } \alpha-2 \mathrm{a}(5 \mathrm{MU}, 1 / 2 \mathrm{~d})\end{array}$ \\
\hline Guan LJ 2006 & 32 & RCT & 2 & $24 w$ & $6 \mathrm{~m}$ & $\begin{array}{l}\text { 1. PEG-IFNa-2a }(180 \mathrm{ug} / \mathrm{w}) \\
\text { 2. Conventional IFNa-2b }(5 \mathrm{MU}, 1 / 2 \mathrm{~d})\end{array}$ \\
\hline Shi XF 2006 & 27 & NRCT & 1 & $6 \mathrm{~m}$ & 0 & 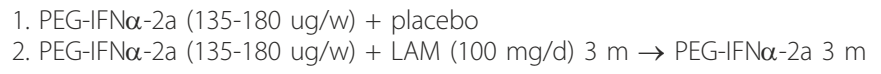 \\
\hline
\end{tabular}


Table 2 Patient selection criteria of studies included in the meta-analysis

\begin{tabular}{|c|c|c|}
\hline Study & Inclusion criteria & Exclusion criteria \\
\hline \multirow{6}{*}{$\begin{array}{l}\text { Lau G.K.K. } \\
2005\end{array}$} & 1. Adults & 1. Treatment within $6 \mathrm{~m}$ \\
\hline & 2. $\mathrm{HBsAg}$ positive for $>6 \mathrm{~m}$ and $\mathrm{HBeAg}$ positive & 2. HIV infection, hepatitis C or D \\
\hline & 3. HBV DNA > 500000 copies $/ \mathrm{ml}$ & 3. Decompensated liver disease \\
\hline & 4. Evidence of inflammation on biopsy and 1 ULN $<$ ALT $<10$ ULN & 4. Serious medical or psychiatric illness \\
\hline & & 5. Alcohol or drug use within $1 \mathrm{y}$ \\
\hline & & $\begin{array}{l}\text { 6. Neutrophils }<1500 / \mathrm{mm}^{3} \text {, platelets }<90000 / \mathrm{mm}^{3} \text {, or } \\
\text { creatinine }>1.5 \text { ULN }\end{array}$ \\
\hline \multirow{6}{*}{$\begin{array}{l}\text { Marcellin P. } \\
2004\end{array}$} & 1. Adults & 1. Treatment within $6 \mathrm{~m}$ \\
\hline & 2. $\mathrm{HBsAg}$ positive for $>6 \mathrm{~m}$ and $\mathrm{HBeAg}$ negative & 2. HIV infection, hepatitis C or D \\
\hline & 3. HBV DNA > 100000 copies/ml & 3. Decompensated liver disease \\
\hline & 4. Evidence of inflammation on biopsy and $1 \mathrm{UNL}<\mathrm{ALT}<10 \mathrm{ULN}$ & 4. Serious medical or psychiatric illness \\
\hline & & 5. Alcohol or drug use within $1 \mathrm{y}$ \\
\hline & & $\begin{array}{l}\text { 6. Neutrophils }<1500 / \mathrm{mm}^{3} \text {, platelets }<90000 / \mathrm{mm}^{3} \text {, or } \\
\text { creatinine }>1.5 \text { ULN }\end{array}$ \\
\hline \multirow{6}{*}{$\begin{array}{l}\text { Marcellin P. } \\
2009\end{array}$} & 1. Adults & 1. Treatment within $6 \mathrm{~m}$ \\
\hline & 2. $\mathrm{HBsAg}$ positive for $>6 \mathrm{~m}$ and $\mathrm{HBeAg}$ negative & 2. HIV infection, hepatitis C or D \\
\hline & 3. HBV DNA > 100000 copies/ml & 3. Decompensated liver disease \\
\hline & 4. Evidence of inflammation on biopsy and 1 ULN $<$ ALT $<10$ ULN & 4. Serious medical or psychiatric illness \\
\hline & & 5. Alcohol or drug use within 1 y \\
\hline & & $\begin{array}{l}\text { 6. Neutrophils }<1500 / \mathrm{mm}^{3} \text {, platelets }<90000 / \mathrm{mm}^{3} \text {, or } \\
\text { creatinine }>1.5 \text { ULN }\end{array}$ \\
\hline \multirow{7}{*}{$\begin{array}{l}\text { Chan H.L.Y. }{ }^{1} \\
2005\end{array}$} & 1. 18-65 years old & $\begin{array}{l}\text { 1. Decompensated liver disease or a history of interferon or } \\
\text { antiviral agent use }\end{array}$ \\
\hline & 2. $\mathrm{HBs} A g$ positive for $>6 \mathrm{~m}$ and $\mathrm{HBeAg}$ positive & 2. HIV infection, hepatitis C or D \\
\hline & 3. HBV DNA > 500000 copies $/ \mathrm{ml}$ & 3. History of hepatocellular carcinoma \\
\hline & 4. $1.3 \mathrm{ULN}<\mathrm{ALT}<5$ ULN & 4. Other causes of liver disease \\
\hline & & 5. Serious medical or psychiatric illness \\
\hline & & $\begin{array}{l}\text { 6. Concurrent use of corticosteroid or immunosuppressive } \\
\text { agents }\end{array}$ \\
\hline & & 7. Pregnancy \\
\hline \multirow[t]{7}{*}{$\begin{array}{l}\text { Chan H.L.Y. } \\
2005\end{array}$} & 1. $18-65$ years old & $\begin{array}{l}\text { 1. Decompensated liver disease or a history of interferon or } \\
\text { antiviral agent use }\end{array}$ \\
\hline & 2. $\mathrm{HBsAg}$ positive for $>6 \mathrm{~m}$ and $\mathrm{HBeAg}$ positive & 2. HIV infection, hepatitis C or D \\
\hline & 3. HBV DNA > 500000 copies $/ \mathrm{ml}$ & 3. History of hepatocellular carcinoma \\
\hline & 4. 1.3 ULN $<$ ALT $<5$ ULN & 4. Other causes of liver disease \\
\hline & & 5. Serious medical or psychiatric illness \\
\hline & & $\begin{array}{l}\text { 6. Concurrent use of corticosteroid or immunosuppressive } \\
\text { agents }\end{array}$ \\
\hline & & 7. Pregnancy \\
\hline \multirow{7}{*}{$\begin{array}{l}\text { Janssen HLA } \\
2005\end{array}$} & 1. $\geq 16$ years old & 1. Antiviral or immunosuppressive therapy within $6 \mathrm{~m}$ \\
\hline & $\begin{array}{l}\text { 2. HBsAg positive for }>6 \mathrm{~m} \text { and } \mathrm{HBeAg} \text { positive on two occasions } \\
\text { within } 8 \mathrm{~W} \text { of randomization }\end{array}$ & 2. HIV infection, hepatitis C or D \\
\hline & $\begin{array}{l}\text { 3. Evidence of inflammation by two measurements of ALT }>2 \text { ULN } \\
\text { within } 8 \mathrm{w} \text { of randomization }\end{array}$ & 3. Advanced liver disease or carcinoma \\
\hline & & $\begin{array}{l}\text { 4. Serious medical or psychiatric illness, or uncontrolled thyroid } \\
\text { disease }\end{array}$ \\
\hline & & 5. Substance abuse within 2 y \\
\hline & & 6. Pregnancy or inadequate contraception \\
\hline & & $\begin{array}{l}\text { 7. Leucocytes } \leq 3000 / \mathrm{mm}^{3} \text {, neutrophils } \leq 1800 / \mathrm{mm}^{3} \text {, or } \\
\text { platelets } \leq 100000 / \mathrm{mm}^{3}\end{array}$ \\
\hline
\end{tabular}


Table 2 Patient selection criteria of studies included in the meta-analysis (Continued)

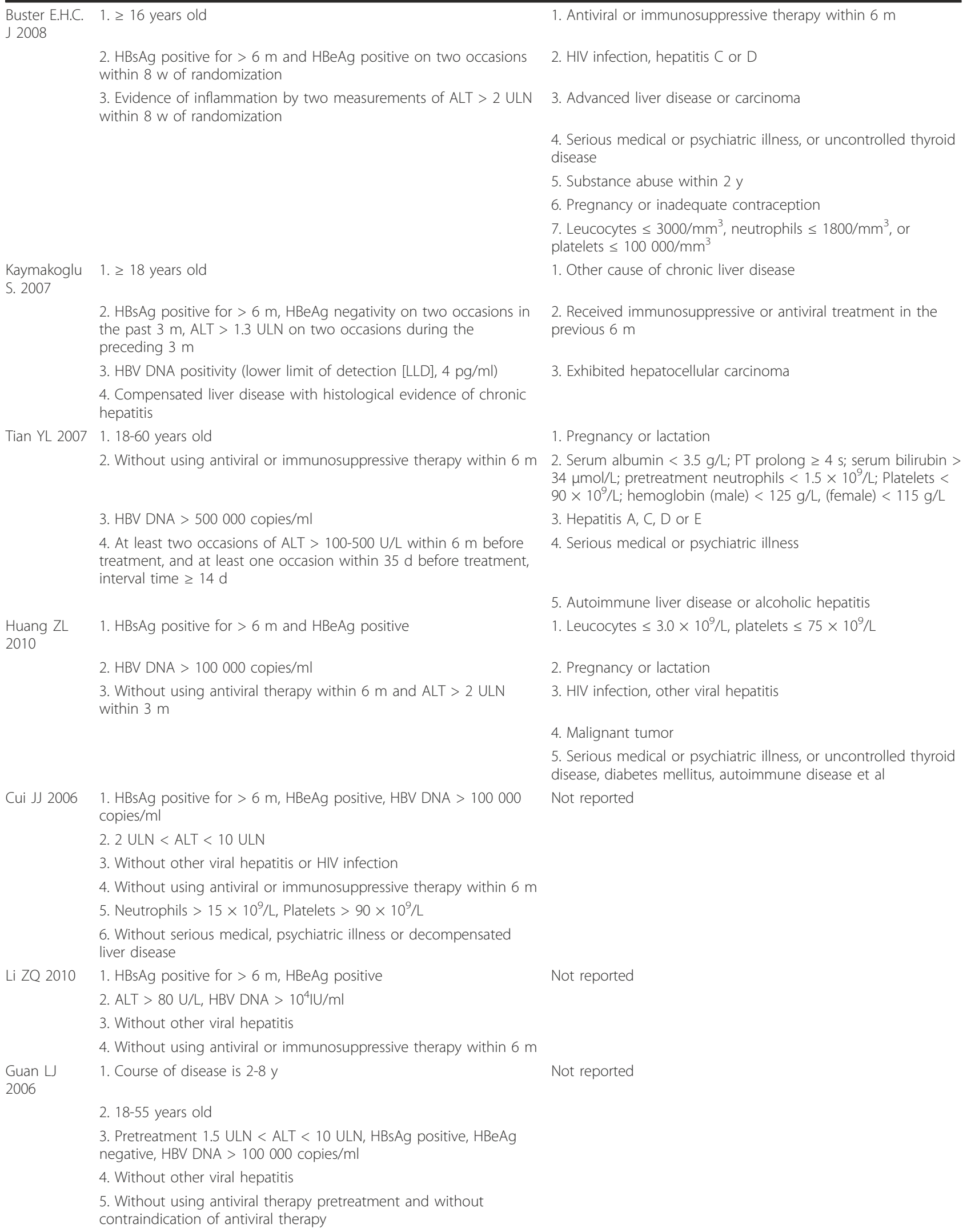

3. Evidence of inflammation by two measurements of ALT $>2$ ULN 3 . Advanced liver disease or carcinoma

Cui JJ 2006 1. HBsAg positive for $>6$ m, HBeAg positive, HBV DNA > 100000 copies $/ \mathrm{ml}$

2. 2 ULN $<$ ALT $<10$ ULN

3. Without other viral hepatitis or HIV infection

4. Without using antiviral or immunosuppressive therapy within $6 \mathrm{~m}$ 5. Neutrophils $>15 \times 10^{9} / \mathrm{L}$, Platelets $>90 \times 10^{9} / \mathrm{L}$

6. Without serious medical, psychiatric illness or decompensated liver disease

Li ZQ 2010 1. HBsAg positive for > 6 m, HBeAg positive

2. $A L T>80 \mathrm{U} / \mathrm{L}, \mathrm{HBV} D N A>10^{4} \mathrm{IU} / \mathrm{ml}$

3. Without other viral hepatitis

4. Without using antiviral or immunosuppressive therapy within $6 \mathrm{~m}$

Guan $\sqcup J \quad$ 1. Course of disease is $2-8 y$

2006

1. Antiviral or immunosuppressive therapy within $6 \mathrm{~m}$

2. HIV infection, hepatitis $C$ or $D$

4. Serious medical or psychiatric illness, or uncontrolled thyroid disease

5. Substance abuse within 2 y

6. Pregnancy or inadequate contraception

7. Leucocytes $\leq 3000 / \mathrm{mm}^{3}$, neutrophils $\leq 1800 / \mathrm{mm}^{3}$, or platelets $\leq 100000 / \mathrm{mm}^{3}$

1. Other cause of chronic liver disease

2. Received immunosuppressive or antiviral treatment in the previous $6 \mathrm{~m}$

3. Exhibited hepatocellular carcinoma

1. Pregnancy or lactation

2. Serum albumin $<3.5 \mathrm{~g} / \mathrm{L}$; PT prolong $\geq 4$ s; serum bilirubin $>$ $34 \mathrm{\mu mol} / \mathrm{L}$; pretreatment neutrophils $<1.5 \times 10^{9} / \mathrm{L}$; Platelets $<$

$90 \times 10^{9} / \mathrm{L}$; hemoglobin (male) $<125 \mathrm{~g} / \mathrm{L}$, (female) $<115 \mathrm{~g} / \mathrm{L}$

3. Hepatitis A, C, D or E

4. Serious medical or psychiatric illness

5. Autoimmune liver disease or alcoholic hepatitis

1. Leucocytes $\leq 3.0 \times 10^{9} / \mathrm{L}$, platelets $\leq 75 \times 10^{9} / \mathrm{L}$

2. Pregnancy or lactation

3. HIV infection, other viral hepatitis

4. Malignant tumor

5. Serious medical or psychiatric illness, or uncontrolled thyroid disease, diabetes mellitus, autoimmune disease et al

Not reported

Not reported

Not reported
2. $18-55$ years old
3. Pretreatment $1.5 \mathrm{ULN}<\mathrm{ALT}<10 \mathrm{ULN}, \mathrm{HBs} A g$ positive, HBeAg
4. Without other viral hepatitis
5. Without using antiviral therapy pretreatment and without contraindication of antiviral therapy negative, HBV DNA > 100000 copies/ml 
Table 2 Patient selection criteria of studies included in the meta-analysis (Continued)

\begin{tabular}{|c|c|c|}
\hline \multirow[t]{6}{*}{ Shi XF 2006} & 1. $16-60$ years old & 1. Decompensated liver disease \\
\hline & $\begin{array}{l}\text { 2. Without using antiviral therapy within } 6 \mathrm{~m} \text { or virological relapse } \\
\text { or YMDD mutation after more than } 1 \text { y LAM treatment }\end{array}$ & 2. Serious medical or psychiatric illness \\
\hline & 3. HBsAg positive, HBeAg positive & 3. Neutrophils $<1500 / \mathrm{mm}^{3}$, Platelets $\leq 70000 / \mathrm{mm}^{3}$ \\
\hline & $\begin{array}{l}\text { 4. HBV DNA > } 100000 \text { copies/ml, or relapse (increase } 2 \log 10 \\
\text { copies/ml) }\end{array}$ & 4. Hepatitis A, C, D or E \\
\hline & 5. Serum total bilirubin $<40 \mathrm{umol} / \mathrm{L}$ & 5. alcohol or drugs abuse within 1 y \\
\hline & $\begin{array}{l}\text { 6. PEG-IFNa-2a monotherapy group } 2 \text { ULN }<\text { ALT }<10 \text { ULN; PEG- } \\
\text { IFN } \alpha \text {-2a combination with LAM group ALT }<10 \text { ULN }\end{array}$ & \\
\hline
\end{tabular}

HBsAg: hepatitis B surface antigen; HBeAg: hepatitis B e antigen; HBV: hepatitis B virus; ALT: alanine aminotransferase; ULN: upper limit of normal; HIV: human immunodeficiency virus; PEG-IFN $\alpha$-2a: pegylated interferon alpha-2a; LAM: lamivudine; d: days; m: months; y: years.

$P=0.07$, and $\mathrm{OR}=9.41,95 \% \mathrm{CI}(1.18-74.94), P=0.03$, respectively]. The fixed effect model for meta-analysis was used according to the heterogeneity test $\left(\chi^{2}=0.37\right.$, $\mathrm{df}=1, P=0.54, \mathrm{I}^{2}=0 \%$ and $\chi^{2}=0.60, \mathrm{df}=1, P=0.44$, $\mathrm{I}^{2}=0 \%$, respectively).

Similarly, HBsAg seroconversion rate was $2.4 \%$ in the combination therapy group and $0 \%$ in the LAM monotherapy group. Heterogeneities were assessed and no concern was found $\left(\chi^{2}=0.19, \mathrm{df}=1, P=0.67, \mathrm{I}^{2}=\right.$ $0 \%$ ), thus allowing the use of a fixed effect model for meta-analysis. The difference reached statistical significance $[\mathrm{OR}=12.37,95 \% \mathrm{CI}(1.60-95.44), P=0.02$ ] [Figure 4].

PEG-IFN $\alpha$ monotherapy vs. IFN $\alpha$ monotherapy

The rates of both HBsAg clearance and HBsAg seroconversion in the PEG-IFN $\alpha$ monotherapy group $(7.6 \%$ and $4.1 \%)$ exceeded the IFN $\alpha$ monotherapy group $(0.8 \%$ and $1.4 \%)$. The fixed effect model for meta-analysis was used according to the heterogeneity test $\left(\chi^{2}=0.43, \mathrm{df}=3, P\right.$ $=0.93, \mathrm{I}^{2}=0 \%$ and $\chi^{2}=0.02, \mathrm{df}=1, P=0.89, \mathrm{I}^{2}=0 \%$, respectively). Significant difference in HBsAg clearance rates was observed between the two groups $[\mathrm{OR}=4.95$,

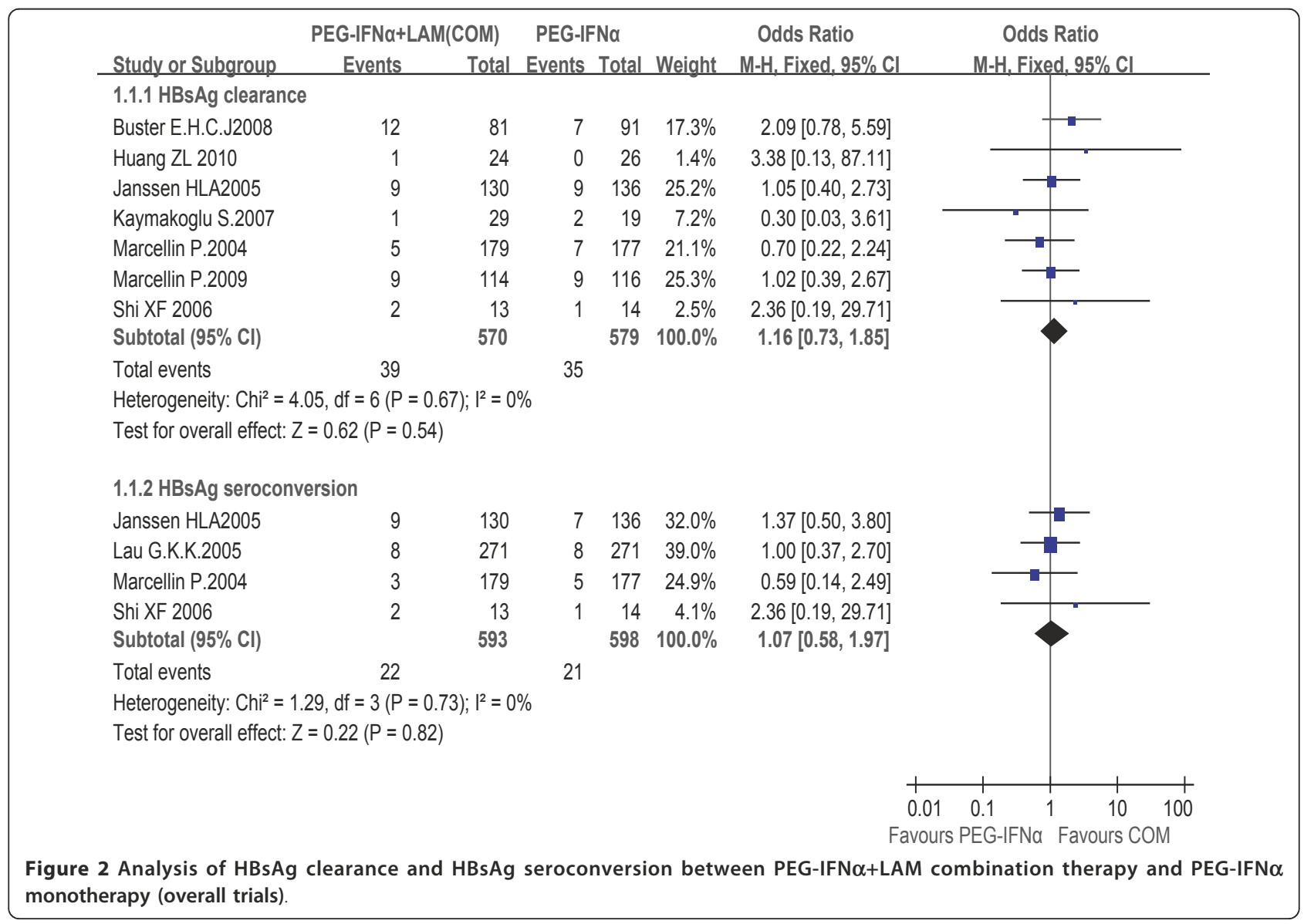




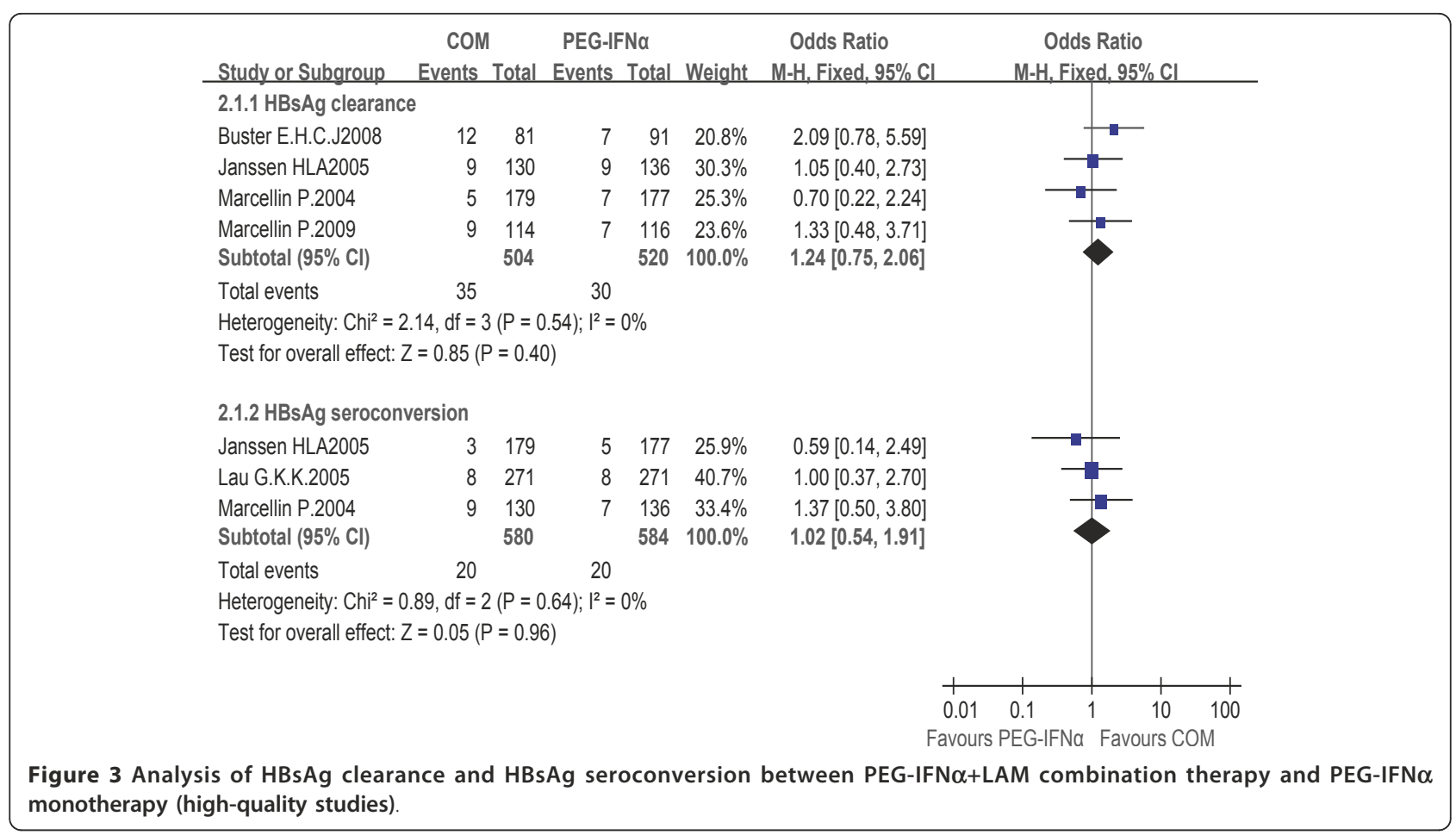

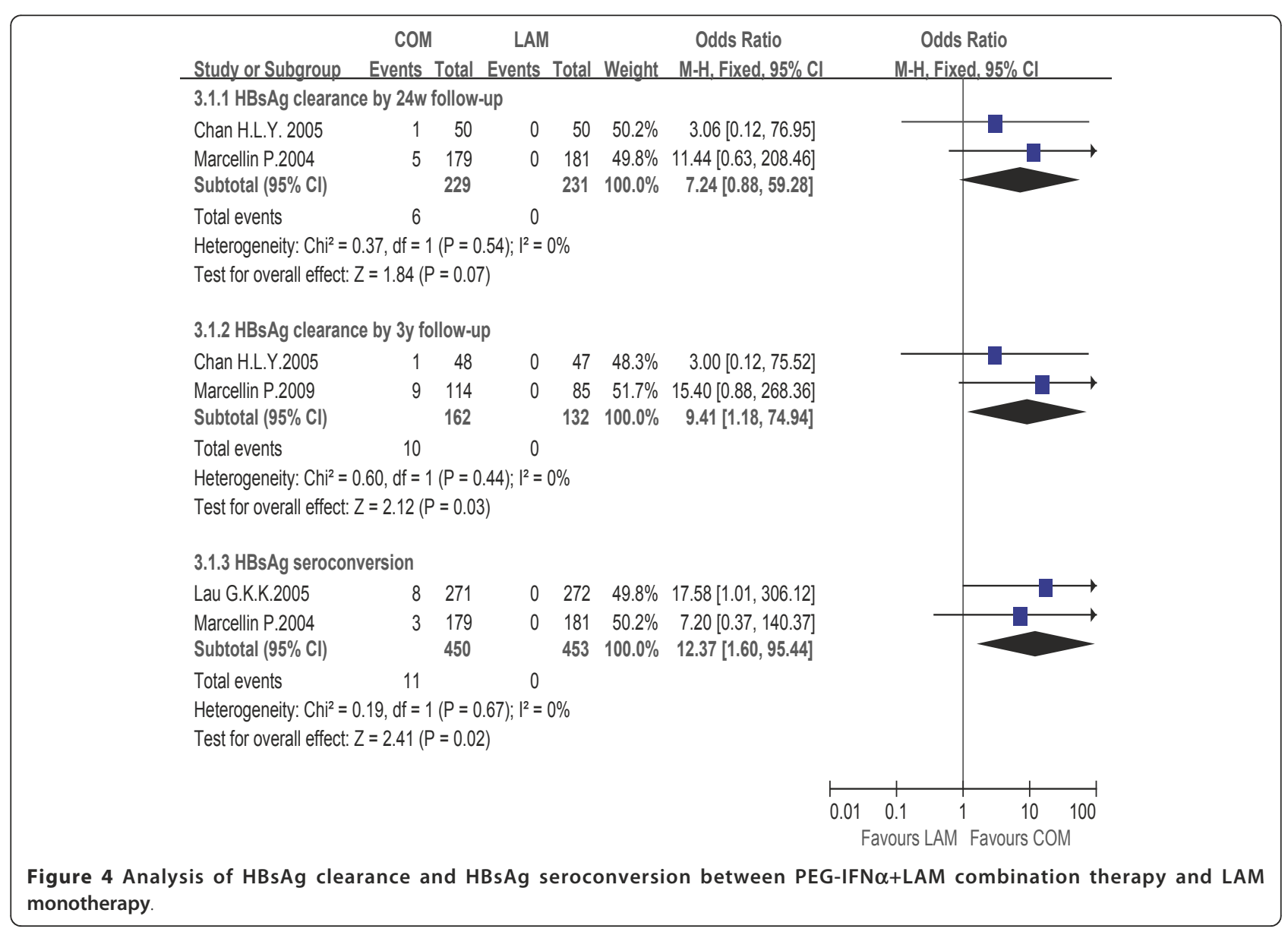




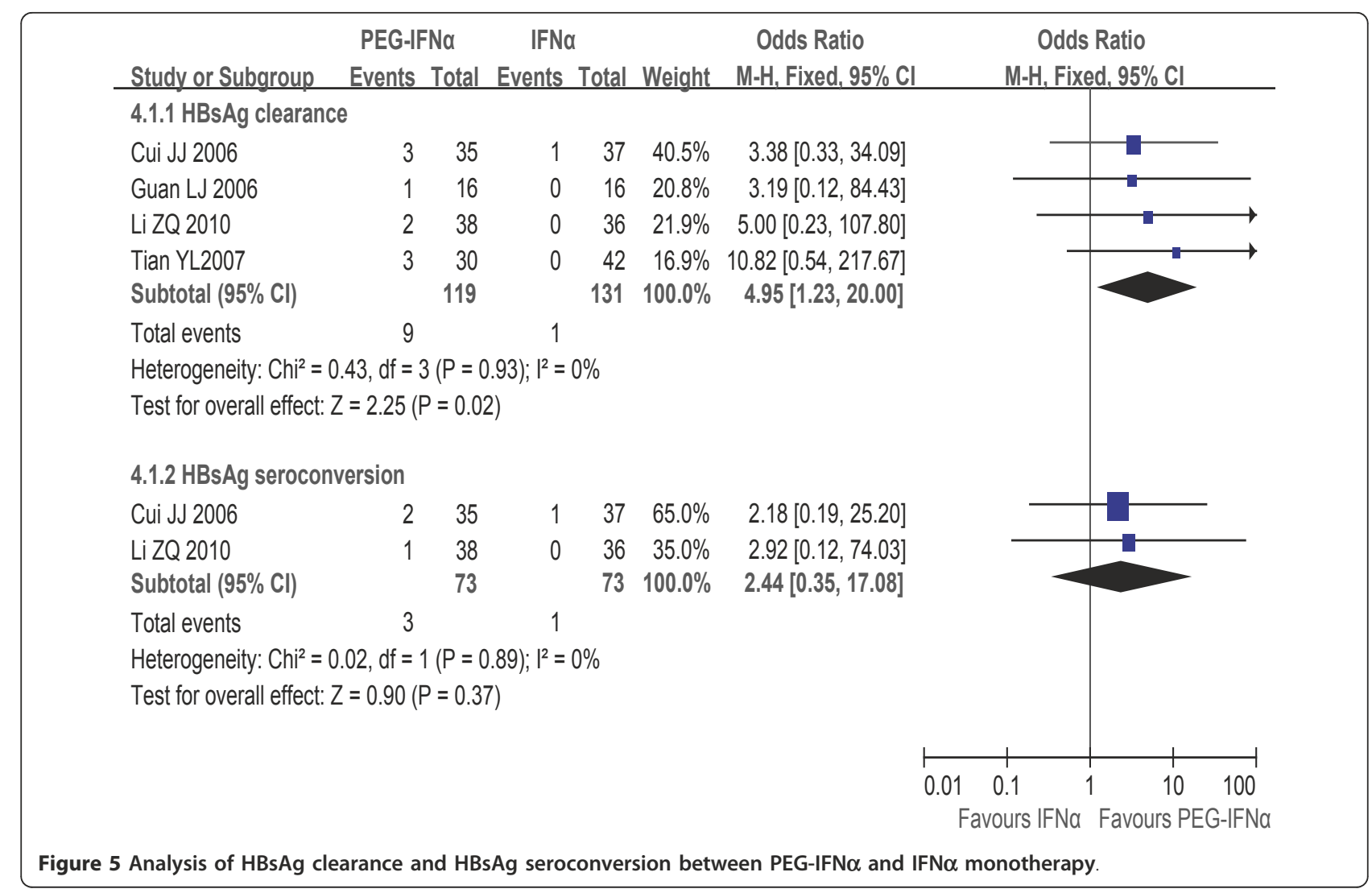

95\% CI (1.23-20.00), $P=0.02$ ], but not in seroconversion rates $[\mathrm{OR}=2.44,95 \% \mathrm{CI}(0.35-17.08), P=0.37]$ [Figure 5].

\section{PEG-IFN $\alpha$ monotherapy vs. LAM monotherapy}

HBsAg seroconversion rate was $2.9 \%$ in PEG-IFNa monotherapy group and $0 \%$ in LAM monotherapy group. The fixed effect model for meta-analysis was used according to the heterogeneity test $\left(\chi^{2}=0.04, \mathrm{df}=\right.$ $\left.1, P=0.84, \mathrm{I}^{2}=0 \%\right)$. The difference between the two groups reached statistical significance $[\mathrm{OR}=14.59,95 \%$ CI (1.91-111.49), $P=0.01$ ] [Figure 6].

\section{Publication bias}

An assessment of publication bias was conducted using funnel plots. Evidences of publication bias based on the funnel plots were found in comparison of combination therapy with PEG-IFN $\alpha$ monotherapy on HBsAg clearance and HBsAg seroconversion only among high-

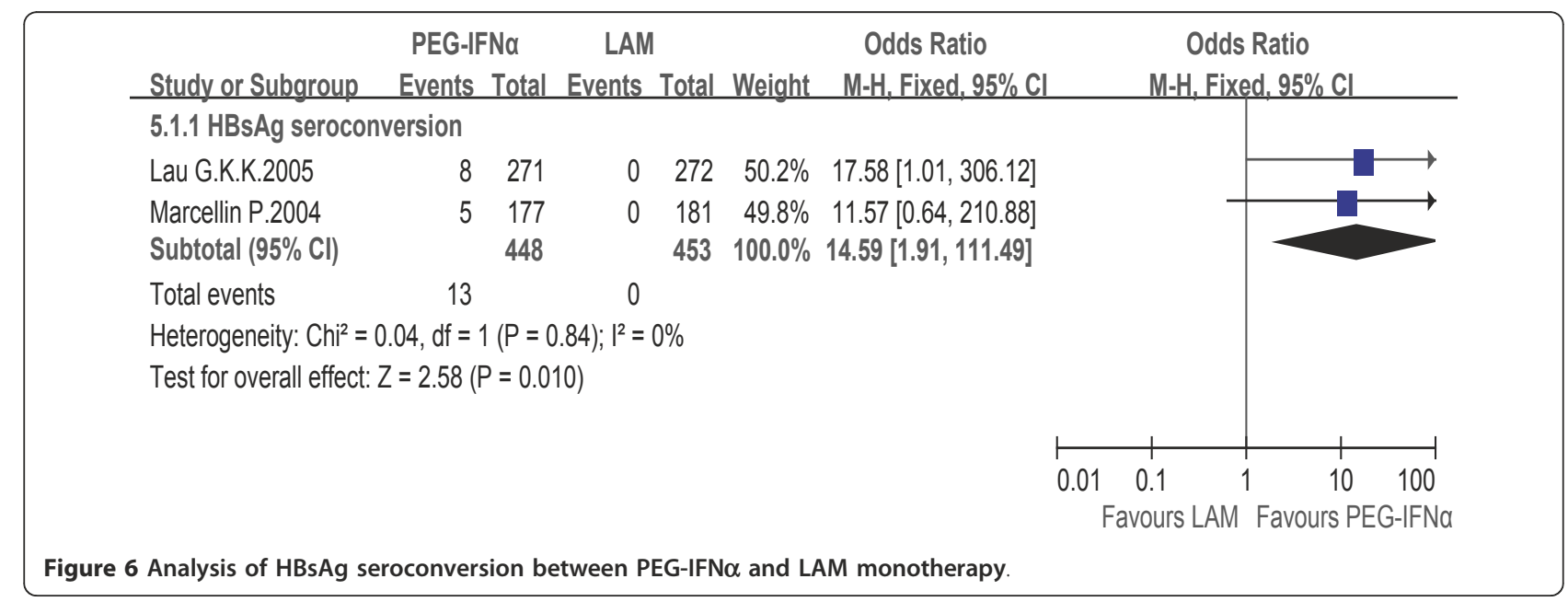


quality studies [Figure 7]. There was no apparent publication bias in the other comparison groups.

\section{Discussion}

Currently there are two kinds of antiviral agents for the treatment of $\mathrm{CHB}$ patients, interferon (conventional or pegylated interferon) and nucleos(t)ide analogues (lamivudine, telbivudine, adefovir, entecavir, and tenofovir). It has been documented that interferon is more efficient than nucleos(t)ide analogues on HBeAg clearance and HBeAg seroconversion. However, the use of interferon was restricted because of the inconvenience of injection and risk of adverse reactions. Nucleos(t)ide analogues were more convenient to use and strongly suppressed HBV DNA replication. However, nucleos(t)ide analogues had the disadvantages of developing resistance and there is no established course of treatment. Combination therapy with interferon and nucleos(t)ide analogues was prompted in order to enhance the therapeutic efficacy.
HBsAg clearance or seroconversion, which might predict intrahepatic covalently closed circular DNA (cccDNA) reduction, is the ultimate outcome of antiviral therapy. However, there are not sufficient studies involved in this objective. Compared with any single study, meta-analysis has increased power for statistical tests, and increased precision for confidence intervals, because the conclusions often reflect a broad spectrum of patients and study characteristics, and the results are more generalizable than a single study. Our study is the first to analyse PEG-IFN $\alpha$-based therapy for CHB treatment focusing on HBsAg clearance or seroconversion, pooling data from all pertinent clinical-controlled trials into meta-analysis. This analysis is to achieve evidencebased conclusions on the matter, to resolve the controversy over the selection of antiviral regimens, and to be further referred to by future clinical investigations.

In this analysis, we found that PEG-IFN $\alpha$ monotherapy and combination with PEG-IFN $\alpha$ and LAM obtained comparable efficacies, achieving similar rates of $\mathrm{HBsAg}$

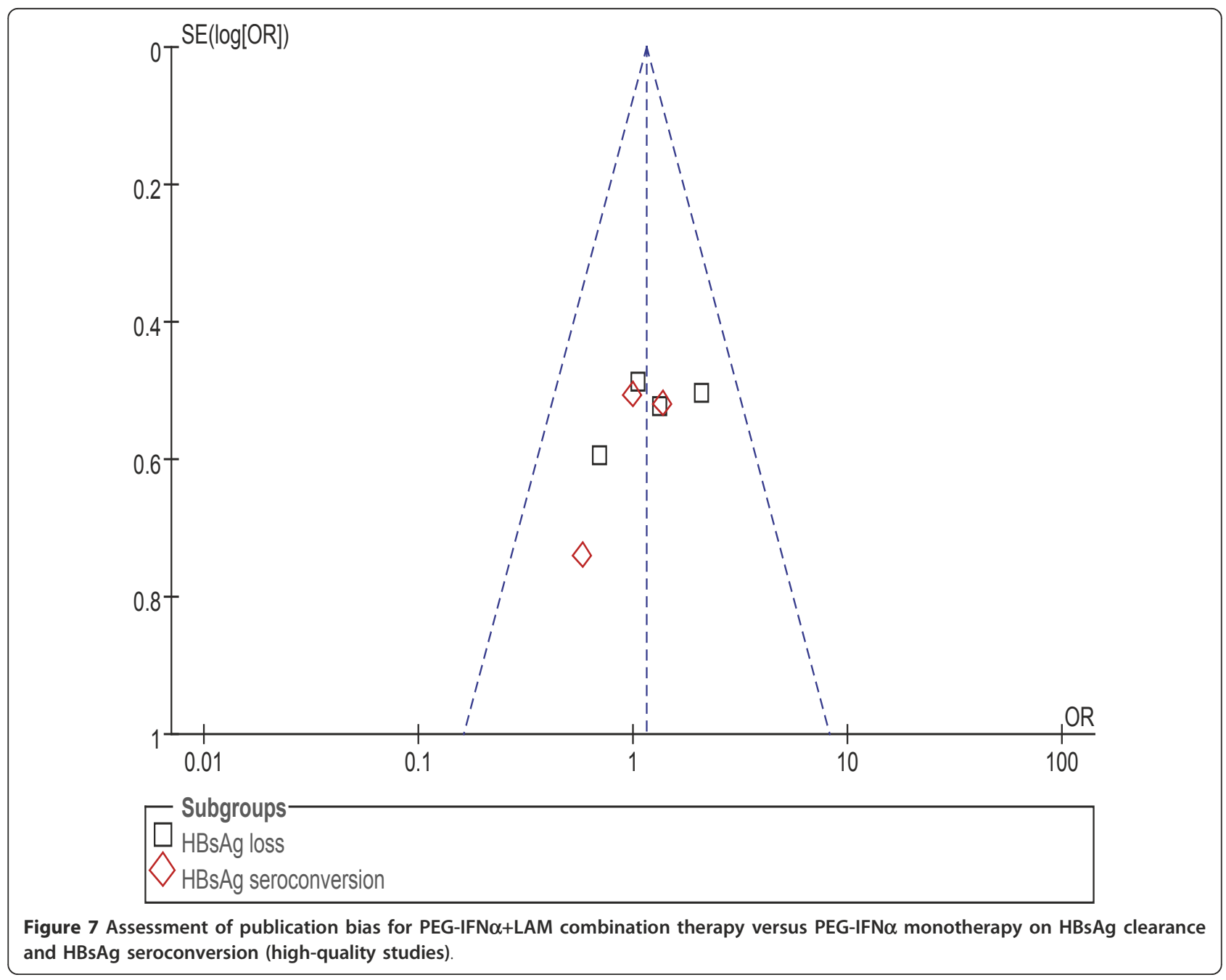


clearance and seroconversion $(P=0.54$ and 0.82 respectively) [15-17,28-32]. We also set up subgroups for metaanalysis according to follow-up terms, but no significant difference was obtained. Thus, the addition of LAM to PEG-IFN $\alpha$ regimen could not promote the clinical cure in defined course of treatment but brought heavy financial burden to patients. Up to date, there was seldom reports of randomized controlled clinical trials based on combination therapy with PEG-IFN $\alpha$ and other nucleos(t)ide analogues. Paola Piccolo et al [33] performed a multicenter randomized controlled trial, in which a total of 60 patients were included and efficacies of PEG-IFN $\alpha$ monotherapy and combination therapy with PEG-IFN $\alpha$ and adefovir dipivoxil were compared. Only one patient (3.3\%) in the combination therapy group obtained HBsAg clearance during $24 \mathrm{w}$ follow-up. The combination of PEG-IFN $\alpha$ with more potential nucleos(t)ide analogues such as entecavir or tenofovir needs to be further investigated in large, randomized studies. Nucleos(t)ide analogues typically demand to be continued indefinitely to maintain viral suppression, without a clearly defined endpoint for stopping treatment. This approach increases the risk of antiviral resistance, and the safety of long-term therapy with nucleos $(\mathrm{t})$ ide analogues also needs to be explored.

Higher rates of HBsAg clearance and seroconversion were observed in patients receiving the combination regimen as compared with those receiving LAM monotherapy [15-19]. Increasing HBsAg loss rate was only obtained by long-term follow-up $[17,19]$. Patients treated with PEG-IFN $\alpha$ monotherapy achieved higher rate of HBsAg seroconversion as compared with those treated with LAM monotherapy $[15,16]$. The difference in HBsAg clearance or seroconversion rates might relate to antiviral mechanisms of the agents. There are two mechanisms of interferon action: (i) direct antiviral effect by suppressing synthesis of viral DNA and by activating antiviral enzymes; and (ii) magnification of the cellular immune response against hepatocytes infected with HBV [34]. PEG-IFNa-based therapy exerts persistently an immunomodulatory activity to produce antibodies to HBV antigens and sustained virological response. Otherwise, nucleos(t)ide analogues, such as LAM, suppresses HBV replication by inhibiting the reverse transcriptase activity of HBV polymerase. Only a few patients sustained the response and HBsAg loss after 6 months of treatment discontinuation $[35,36]$. The same conclusions were drawn from comparisons between PEG-IFN $\alpha$ and adefovir dipivoxil or entecavir [37-39]. Complementary activity of immunoregulation and sustained suppression of HBV replication in CHB patients can be achieved by combination of PEG-IFN $\alpha$ and nucleos(t)ide analogues, which can initiate specific immune response to bring about HBsAg elimination and seroconversion.
Clearance rate of HBsAg was significantly higher in patients receiving PEG-IFN $\alpha$ monotherapy than in those receiving conventional IFN $\alpha$ monotherapy [20-23], which might attribute to their different pharmacokinetics. Conventional IFN $\alpha$ was administered once every other day, and it reached peak concentration rapidly in the blood and got lower at intervals. PEG-IFN $\alpha$ was used once per week, with its effective blood concentration persisting for a week [24]. Currently, the updated therapeutic guidelines on management of $\mathrm{CHB}$ suggest that PEG-IFN $\alpha$ should be administered instead of conventional IFNa to improve antiviral response.

Three studies providing 3-year follow-up data were included for the analysis of HBsAg clearance rate in our study. Higher rate of HBsAg clearance was observed in patients with the combination therapy as compared with those using LAM monotherapy $(P=$ 0.03 ), but no significant difference was observed after short-term follow-up investigation $(P=0.07)$. The sustained long-term efficacy of PEG-IFN $\alpha$-based therapy was reflected by increased proportion of patients achieving HBsAg clearance during follow-up $[17,30,40,41]$. It may attribute to the effect of PEGIFN $\alpha$ since HBsAg clearance was rarely obtained in patients treated with lamivudine, adefovir, entecavir or tenofovir [42-44]. It also indicated that long-term follow-up is necessary to achieve evidence of optimized antiviral strategy.

Our study contains several limitations. Firstly, although no difference was found between the highquality studies and the overall trials in the comparison of the combination therapy group and PEG-IFN $\alpha$ monotherapy group, the low-quality studies in our analysis, especially those in Chinese publications, which lacked randomization, may weaken our conclusions. Secondly, while this study focused on PEG-IFNa and LAM combination therapy, combinations of PEG-IFNa with other potent antiviral agents need to be further explored, which was limited by deficient data currently available. Finally, the absence of adequate controlled trials precluded our analysis on the subsets of PEGIFN $\alpha-2 a$ or $2 b$, as well as $\mathrm{HBeAg}$ positive or negative CHB patients.

\section{Conclusions}

PEG-IFN $\alpha$ facilitated HBsAg clearance or seroconversion in CHB patients. PEG-IFN $\alpha$-based therapy was more effective than LAM monotherapy in achieving HBsAg clearance or seroconversion for both HBeAgpositive and HBeAg-negative $\mathrm{CHB}$ patients. There was no significant difference in HBsAg clearance or seroconversion between the combination therapy and PEGIFN $\alpha$ monotherapy. PEG-IFN $\alpha$ was obviously superior to conventional IFNa in HBsAg clearance, but not in 
HBsAg seroconversion. Although PEG-IFN $\alpha$ produced significantly higher rates of HBsAg clearance and seroconversion, the absolute change in the proportion of HBsAg clearance and seroconversion was low (about 3$6 \%)$. Therefore, additional interventions are needed to improve positive outcomes.

\section{List of Abbreviations}

IFNa: interferon alpha; PEG-IFNa: pegylated interferon alpha, peginterferon $a$; LAM: Iamivudine; CHB: chronic hepatitis B; HBsAg: hepatitis B surface antigen; HBeAg: hepatitis B e antigen; HBV: hepatitis B virus; cccDNA: covalently closed circular DNA; ALT: alanine aminotransferase; ULN: upper limit of normal; HIV: human immunodeficiency virus; OR: odds ratio; $\mathrm{Cl}$ : confidence intervals; $x^{2}$ : chi-square; $l^{2}$ : I square; RCT: randomized controlled trial; NRCT: nonrandomized controlled trial; d: days; w: weeks; m: months; $y$ : years.

\section{Acknowledgements}

We thank Prof. Qing-bao Tian (Public Health College of Hebei Medical University, China) and Prof. Jun Yu (Institute of Digestive Disease and Department of Medicine and Therapeutics, The Chinese University of Hong Kong, Hong Kong) for their advices on this study.

\section{Author details}

${ }^{1}$ Department of Traditional and Western Medical Hepatology, Third Hospital of Hebei Medical University, Shijiazhuang, China. ${ }^{2}$ Department of Infectious diseases, Nanjing 81 Hospital, Nanjing 210002, China.

\section{Authors' contributions}

YN designed the research; WL and WR operated the literature retrieval, trial selection and data extraction; WL, MW, LK and YZ analyzed data; WL, MW, LK, WR and YZ wrote the paper. All authors read and approved the final manuscript.

\section{Competing interests}

The authors report no conflicts of interest. The authors alone are responsible for the content and writing of the paper.

Received: 5 January 2011 Accepted: 9 June 2011 Published: 9 June 2011

\section{References}

1. Wilkins T, Zimmerman D, Schade RR: Hepatitis B: Diagnosis and Treatment. Am Fam Physician 2010, 81:965-972.

2. Papatheodoridis GV, Manolakopoulos S, Archimandritis AJ: Current treatment indications and strategies in chronic hepatitis B virus infection. World J Gastroenterol 2008, 14:6902-6910.

3. Liaw YF, Chu CM: Hepatitis B virus infection. Lancet 2009, 373:582-592.

4. Zoulim F, Perrillo R: Hepatitis B: Reflections on the current approach to antiviral therapy. J Hepatol 2008, 48:S2-S19.

5. Hsu YS, Chien RN, Yeh CT, Sheen IS, Chiou HY, Chu CM, Liaw YF: Longterm outcome after spontaneous $\mathrm{HBeAg}$ seroconversion in patients with chronic hepatitis B. Hepatology 2002, 35:1522-1527.

6. Niederau C, Heintges T, Lange S, Goldmann G, Niederau CM, Mohr L, Häussinger D: Long-term follow-up of HBeAg-positive patients treated with interferon alfa for chronic hepatitis B. N Engl J Med 1996, 334:1422-1427.

7. Hadziyannis SJ, Vassilopoulos D: Hepatitis B e antigen-negative chronic hepatitis B. Hepatology 2001, 34:617-624.

8. Brunetto MR, Oliveri F, Coco B, Leandro G, Colombatto P, Gorin JM, Bonino F: Outcome of anti-HBe positive chronic hepatitis B in alphainterferon treated and untreated patients: a long term cohort study. J Hepatol 2002, 36:263-270.

9. Lampertico P, Del Ninno E, Vigano M, Romeo R, Donato MF, Sablon E, Morabito A, Colombo M: Long-term suppression of hepatitis B e antigennegative chronic hepatitis B by 24-month interferon therapy. Hepatology 2003, 37:756-763.

10. Lok AS, McMahon BJ: Chronic hepatitis B: update of recommendations. Hepatology 2004, 39:857-861.
11. de Franchis R, Hadengue A, Lau G, Lavanchy D, Lok A, McIntyre N, Mele A, Paumgartner G, Pietrangelo A, Rodés J, Rosenberg W, Valla D, EASL Jury: EASL International Consensus Conference on Hepatitis B, 13-14 September, 2002, Geneva, Switzerland: consensus statement (long version). J Hepatol 2003, 39:S3-S25.

12. Lok AS, McMahon BJ: Chronic hepatitis B. Hepatology 2007, 45:507-539.

13. Fattovich G, Giustina G, Sanchez-Tapias J, Quero C, Mas A, Olivotto PG, Solinas A, Almasio P, Hadziyannis S, Degos F, de Moura MC, Krogsgaard K, Pantalena M, Realdi G, Corrocher R, Schalm SW: Delayed clearance of serum HBsAg in compensated cirrhosis B: relation to interferon alpha therapy and disease prognosis. Am J Gastroentero/ 1998, 93:896-900.

14. Lin SM, Yu ML, Lee CM, Chien RN, Sheen IS, Chu CM, Liaw YF: Interferon therapy in $\mathrm{HBeAg}$ positive chronic hepatitis reduces progression to cirrhosis and hepatocellular carcinoma. J Hepatol 2007, 46:45-52.

15. Lau GK, Piratvisuth T, Luo KX, Marcellin P, Thongsawat S, Cooksley G, Gane E, Fried MW, Chow WC, Paik SW, Chang WY, Berg T, Flisiak R, McCloud P, Pluck N, Peginterferon Alfa-2a HBeAg-Positive Chronic Hepatitis B Study Group: Peginterferon alfa-2a, Lamivudine, and the combination for HBeAg-positive chronic hepatitis B. N Engl J Med 2005, 352:2682-2695.

16. Marcellin $\mathrm{P}$, Lau GK, Bonino F, Farci $\mathrm{P}$, Hadziyannis $\mathrm{S}$, Jin R, Lu ZM, Piratvisuth T, Germanidis G, Yurdaydin C, Diago M, Gurel S, Lai MY, Button P, Pluck N, Peginterferon Alfa-2a HBeAg-Negative Chronic Hepatitis B Study Group: Peginterferon alfa-2a alone, Lamivudine alone, and the two in combination in patients with HBeAg-negative chronic hepatitis B. N Engl J Med 2004, 351:1206-1217.

17. Marcellin P, Bonino F, Lau GK, Farci P, Yurdaydin C, Piratvisuth T, Jin R, Gurel S, Lu ZM, Wu J, Popescu M, Hadziyannis S, Peginterferon alfa-2a in HBeAg-negative Chronic Hepatitis B Study Group: Sustained Response of Hepatitis B e Antigen-Negative Patients 3 Years After Treatment with Peginterferon Alfa-2a. Gastroenterology 2009, 136:2169-2179.

18. Chan HL, Leung NW, Hui AY, Wong WW, Liew CT, Chim AM, Chan FK, Hung LC, Lee YT, Tam JS, Lam CW, Sung JJ: Randomized, Controlled Trial of Combination Therapy for Chronic Hepatitis B: Comparing Pegylated Interferon-2b and Lamivudine with Lamivudine Alone. Ann Intern Med 2005, 142:240-250.

19. Chan HL, Hui AY, Wong WW, Chim AM, Wong ML, Sung JJ: Long-Term Follow-Up of Peginterferon and Lamivudine Combination Treatment in HBeAg-Positive Chronic Hepatitis B. Hepatology 2005, 41:1357-1364.

20. Tian YL, Zhao W, Shen L, Liu W, Chang JB, Fang ZX, Yin WW, Wang L, Sun $X B$, Yang YJ: Pegylated interferon alpha $2 a$ in treating chronic hepatitis B patients. Zhonghua Gan Zang Bing Za Zhi 2006, 14:806-810.

21. Cui JJ, Fan P, Jiang XL, Xu C, He Q, Zhou BP: Efficacy and safety of peginterferon alfa-2a treatment in patients with $\mathrm{HBeAg-positive} \mathrm{chronic}$ hepatitis B. Chinese J Exp Clin Virol 2006, 20:331-333.

22. Li ZQ, Sun CY, Wu SH, Yu ZJ, Jiang HQ: Evaluation of Efficacy of Peginterferona-2a Therapy in patients with $\mathrm{HBeAg}$ Positive Chronic Hepatitis B. Journal of Medical Forum 2010, 31:51-53.

23. Guan $\sqcup$, Fan RS, Chen JF: Pegylated interferon in treating HBeAg negative chronic hepatitis B patients. Clinical Medical Journal of China 2006, 13:959.

24. Lau GK: HBeAg-positive chronic hepatitis B: why do I treat my patients with pegylated interferon. Liver International 2009, 29:125-129.

25. Lok AS, McMahon BJ: Chronic hepatitis B: update 2009. Hepatology 2009, 50:661-662.

26. European Association for the Study of the Liver: EASL Clinical Practice Guidelines: Management of chronic hepatitis B. J Hepatol 2009, 50:227-242.

27. Jadad AR, Moore RA, Carroll D, Jenkinson C, Reynolds DJ, Gavaghan DJ, McQuay HJ: Assessing the quality of reports of randomized clinical trials: is blinding necessary? Control Clin Trials 1996, 17:1-12.

28. Janssen $\mathrm{HL}$, van Zonneveld M, Senturk H, Zeuzem S, Akarca US, Cakaloglu Y, Simon C, So TM, Gerken G, de Man RA, Niesters HG, Zondervan P, Hansen B, Schalm SW, HBV 99-01 Study Group; Rotterdam Foundation for Liver Research: Pegylated interferon alfa-2b alone or in combination with lamivudine for $\mathrm{HBeAg}$-positive chronic hepatitis $\mathrm{B}$ : a randomised trial. Lancet 2005, 365:123-129.

29. Shi XF: The Primary Clinical Study of Peginterferon Alfa-2a in the Treatment the Patients with HBeAg-Positive Chronic Hepatitis B and with Drug Fast to Lamivudine. CNKI: CDMD , 1.2006.176314.

30. Buster EH, Flink HJ, Cakaloglu Y, Simon K, Trojan J, Tabak F, So TM, Feinman SV, Mach T, Akarca US, Schutten M, Tielemans W, van Vuuren AJ, 
Hansen BE, Janssen HL: Sustained HBeAg and HBsAg Loss after Longterm Follow-up of HBeAg-Positive Patients Treated With Peginterferon a-2b. Gastroenterology 2008, 135:459-467.

31. Kaymakoglu S, Oguz D, Gur G, Gurel S, Tankurt E, Ersöz G, Ozenirler $S$, Kalayci C, Poturoglu S, Cakaloglu Y, Okten A: Pegylated Interferon Alfa-2b Monotherapy and Pegylated Interferon Alfa-2b plus Lamivudine Combination Therapy for Patients with Hepatitis B Virus E AntigenNegative Chronic Hepatitis B. Antimicrob Agents Chemother 2007, 51:3020-3022.

32. Huang ZL, Zhao ZX, Deng H, Zhang YF, Lu CR, Gao ZL: A pilot study of peginterferon alfa-2a combined with short-term lamivudine therapy in HBeAg-positive chronic hepatitis B patients. Zhonghua Gan Zang Bing Za Zhi 2010, 18:419-422.

33. Piccolo P, Lenci I, Demelia L, Bandiera F, Piras MR, Antonucci G, Nosotti L, Mari T, De Santis A, Ponti ML, Sorbello O, lacomi F, Angelico M: A randomized controlled trail of pegylated interferon-alpha 2a plus adefovir dipivoxil for hepatitis B e antigen-negative chronic hepatitis B. Antiviral Therapy 2009, 14:1165-1174.

34. Marcellin P, Lada O, Asselah T: Treatment of chronic hepatitis B with the combination of pegylated interferon with lamivudine. Hepatology Research 2007, 37:S55-S61.

35. Shouval $D$, Lai CL, Chang $T$, Cheinquer H, Martin P, Carosi G, Han S, Kaymakoglu S, Tamez R, Yang J, Tenney D, Brett-Smith H: Relapse of hepatitis B in HBeAg-negative chronic hepatitis B patients who discontinued successful entecavir treatment: the case for continuous antiviral therapy. J Hepatol 2009, 50:289-295.

36. Reijnders JG, Perquin MJ, Zhang N, Hansen BE, Janssen HL: Nucleos(t)ide analogues only induce temporary hepatitis $B$ e antigen seroconversion in most patients with chronic hepatitis B. Gastroenterology 2010, 139:491-498.

37. Tang SB, Ke CZ, Kang J, Li D, Chen Y: Investigation of PEG-IFNa-2a treatment of chronic hepatitis $B$ patients who failed to lamivudine therapy. $J$ Cl in Hepatol 2007, 10:311-312.

38. Hou J, Sun J, Xie Q, Li X, Zhang J, Wang Y, Wang H, Jak YL, Chen S, Jia J, Sheng J, Chan H, Wang J, Li M, Jiang M, Popescu M, Sung J: A prospective, randomized study of efficacy and safety of peginterferon alfa-2a versus adefovir dipivoxil in treating lamivudine-resistant $\mathrm{HBeAg-positive} \mathrm{CHB.} \mathrm{J}$ Hepatol 2009, 50:5330

39. Chen XF, Chen XP, Huang J, Chen W-L, Chen R: Comparison of peginterferon alfa-2a versus entecavir in patients with $\mathrm{HBeAg-positive}$ chronic hepatitis B with mildly elevated alanine aminotransferase levels. J Hepatol 2009, 50:S328.

40. Marcellin P, Piratvisuth $T$, Brunetto $M$, Bonino F, Popescu M, Farci P, Yurdaydin C, Wu J, Lau GKK: Virological and biochemical response in patients with $\mathrm{HBeAg}$ negative chronic hepatitis $\mathrm{B}$ treated with peginterferon alfa-2a $(40 \mathrm{kd})$ with or without lamivudine: results of 4-year follow-up. J Hepatol 2008, 48:548.

41. Marcellin P, Piratvisuth T, Brunetto M, Bonino F, Lau GKK, Farci P, Yurdaydin C, Gurel S, Wu J, Popescu M: Increasing rates of HBsAg clearance and seroconversion in patients with $\mathrm{HBeAg}$-negative disease treated with peginterferon alfa- $2 \mathrm{a} \pm$ lamivudine: results of 5 -year posttreatment follow up. J Hepatol 2009, 50:S336.

42. Hadziyannis SJ, Tassopoulos NC, Heathcote EJ, Chang TT, Kitis G, Rizzetto M, Marcellin P, Lim SG, Goodman Z, Ma J, Arterburn S, Xiong S, Currie G, Brosgart CL, Adefovir Dipivoxil 438 Study Group: Long-term therapy with adefovir dipivoxil for HBeAg-negative chronic hepatitis B. N Engl J Med 2005, 352:2673-2681.

43. Lai CL, Shouval D, Lok AS, Chang TT, Cheinquer H, Goodman Z, DeHertogh D, Wilber R, Zink RC, Cross A, Colonno R, Fernandes L, BEHoLD Al463027 Study Group: Entecavir versus lamivudine for patients with HBeAg-negative chronic hepatitis B. N Engl J Med 2006, 354:1011-1020

44. Marcellin P, Heathcote EJ, Buti M, Gane E, de Man RA, Krastev Z, Germanidis G, Lee SS, Flisiak R, Kaita K, Manns M, Kotzev I, Tchernev K, Buggisch P, Weilert F, Kurdas OO, Shiffman ML, Trinh H, Washington MK, Sorbel J, Anderson J, Snow-Lampart A, Mondou E, Quinn J, Rousseau F: Tenofovir disoproxil fumarate versus adefovir dipivoxil for chronic hepatitis B. N Engl J Med 2008, 359:2442-2455.

\section{Pre-publication history}

The pre-publication history for this paper can be accessed here:

http://www.biomedcentral.com/1471-2334/11/165/prepub doi:10.1186/1471-2334-11-165

Cite this article as: Li et al:: Peginterferon alpha-based therapy for chronic hepatitis B focusing on $\mathrm{HBsAg}$ clearance or seroconversion: a meta-analysis of controlled clinical trials. BMC Infectious Diseases 2011 11:165.

\section{Submit your next manuscript to BioMed Central and take full advantage of:}

- Convenient online submission

- Thorough peer review

- No space constraints or color figure charges

- Immediate publication on acceptance

- Inclusion in PubMed, CAS, Scopus and Google Scholar

- Research which is freely available for redistribution

Submit your manuscript at www.biomedcentral.com/submit
Biomed Central 